\title{
¿Solar Monitoring of the NEXRAD WSR-88D Network Using Operational Scan Data
}

\author{
IWAN Holleman, ${ }^{\mathrm{a}}$ Asko HuUsKonen, ${ }^{\mathrm{b}}$ AND BRANDON TAYLOR ${ }^{\mathrm{c}}$

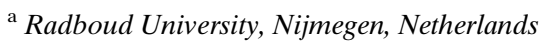 \\ ${ }^{\mathrm{b}}$ Finnish Meteorological Service, Helsinki, Finland \\ ${ }^{\mathrm{c}}$ Centurion Corporation, Norman, Oklahoma
}

(Manuscript received 14 December 2020, in final form 14 October 2021)

\begin{abstract}
Solar monitoring is a method in which solar interferences, recorded during operational scanning of a radar, are used to monitor antenna pointing, identify signal processor issues, track receiver chain stability, and check the balance between horizontal and vertical polarization receive channels. The method is used by EUMETNET to monitor more than 100 radars in 20 European countries and it has been adopted by many national weather services across the world. NEXRAD is a network of 160 similar S-band weather radars (WSR-88Ds), which makes it most suitable for assessing the capability of the solar monitoring method on a continental scale. The NEXRAD level-II data contain radial-by-radial noise power estimates. An increase in this estimate is observed when the antenna points close to the sun. Our decoding software extracts these noise power estimates for the horizontal and vertical receive channels (converted to solar flux units) and other relevant metadata, including azimuth, elevation, observation time, and radar location. Here we present results of analyzing one year of solar monitoring data generated by 142 radars from the contiguous United States. We show monitoring results, geographical maps, and statistical outcomes on antenna pointing, solar fluxes, and differential reflectivity biases. We also assess the quality of the radars by defining a figure of merit, which is calculated from the solar monitoring results. The results demonstrate that the solar method provides great benefit for routine monitoring and harmonization of national and transnational operational radar networks.
\end{abstract}

KEYWORDS: Hydrometeorology; Radars/Radar observations; Weather radar signal processing

\section{Introduction}

Proper adjustment of the antenna pointing is critical for the radar performance. Antenna pointing is further complicated due to the requirement that physical and electrical pointing of the antenna need to coincide, and very few, if any, reference objects besides the sun are available. Proper adjustment of radar elevation using offline solar observations, i.e., using a dedicated scan at high elevations while pointed directly at the sun, may not reliably represent the antenna pointing close to horizon, and observations made close to horizon depend on the reliability of the refraction model (Huuskonen and Holleman 2007). Similarly, the regular monitoring of the radar receiver chains and the differential reflectivity calibration is crucial for successful applications of polarimetric radar (Frech and Hubbert 2020).

Our solar monitoring method is based on using occasional solar interferences recorded by the moving antenna during regular operational scanning of the radar. Solar interferences recorded during a period of time are analyzed together to get the monitoring results. The method has been developed in a series of papers by Huuskonen and Holleman (2007), where they describe how instances of solar interference are used to

Huuskonen: Passed away in June 2021.

\footnotetext{
๑Denotes content that is immediately available upon publication as open access.
}

Corresponding author: Iwan Holleman, iwan.holleman@ru.nl determine the magnitude of antenna bias in azimuth and elevation. Further, Holleman et al. (2010a) developed the formulas to determine the solar flux from these measurements. Also, Huuskonen et al. (2014) and Altube et al. (2015) studied the use of the width of the solar image, while Holleman et al. (2010b) and Huuskonen et al. (2016) described the analysis of the solar differential reflectivity. In addition, Holleman and Huuskonen (2013) developed a model for calculating the refraction of the radio waves, which is consistent with the $k$ model commonly used to calculate the propagation of radio waves through the atmosphere (Bean and Dutton 1966; Doviak and Zrnić 1993). The solar monitoring method is used by the EUMETNET radar program Operational Programme for the Exchange of Weather Radar Information (OPERA) to monitor more than 100 radars in 20 European radar networks (Huuskonen et al. 2018), and it is utilized by many national weather services across the world (e.g., Gabella et al. 2015; Frech and Hubbert 2020). The method is beneficial for harmonizing the networks by providing a means of monitoring and adjusting the antenna pointing and receiver calibration using a common external signal source.

The NEXRAD Weather Surveillance Radar-1988 Doppler (WSR-88D) network is owned jointly by the U.S. Departments of Commerce, Defense, and Transportation. The controlling agencies are the National Weather Service, Air Force Weather Service, and Federal Aviation Administration, respectively. The network consists of 160 similar S-band polarimetric radars, which makes it the largest homogeneous weather radar network in the world, of which 142 radars are situated within the contiguous United States. The original WSR-88D network was installed between 1992 and 1997 
TABLE 1. Example of a volume coverage pattern (VCP-212) and associated waveform types (WFT) for the NEXRAD network. The covered elevations, azimuthal resolution, and solar (azimuthal) resolution are given in degrees.

\begin{tabular}{|c|c|c|c|c|c|c|c|}
\hline Name & $\begin{array}{l}\text { Waveform type } \\
\text { description }\end{array}$ & Covered elevations $\left({ }^{\circ}\right)$ & $\begin{array}{l}\text { Azimuthal } \\
\text { resolution }\left(^{\circ}\right)\end{array}$ & $\begin{array}{l}\text { Surveillance } \\
\text { pulses }\end{array}$ & Doppler pulses & Solar use & $\begin{array}{c}\text { Solar } \\
\text { resolution }\left(^{\circ}\right)\end{array}$ \\
\hline WFT1 & Contiguous surveillance & $0.5,0.9,1.3$ & 0.5 & 15 & 0 & Yes & 1.0 \\
\hline WFT2 & $\begin{array}{l}\text { Contiguous doppler } \\
\text { with ambiguity } \\
\text { resolution }\end{array}$ & $0.5,0.9,1.3$ & 0.5 & 0 & $47-75$ & No & - \\
\hline WFT3 & $\begin{array}{l}\text { Contiguous doppler } \\
\text { without ambiguity } \\
\text { resolution }\end{array}$ & $8,10,12.5,15.6,19.5$ & 1.0 & 0 & $27-44$ & Yes & 1.0 \\
\hline WFT4 & Batch & $1.8,2.1,3.1,4.0,5.1,6.4$ & 1.0 & 3 & $24-39$ & Yes & $0.1-0.2$ \\
\hline
\end{tabular}

(Crum and Alberty 1993; Serafin and Wilson 2000), and upgraded with polarimetric capability between 2010 and 2013 (Saxion et al. 2011).

On the WSR-88Ds, offline solar observations are used to determine the antenna bias, antenna beamwidth, and antenna gain, as described by Ice et al. (2014). They also describe how the differential reflectivity $\left(Z_{\mathrm{DR}}\right)$ bias is obtained using the measured power difference between $\mathrm{H}$ and $\mathrm{V}$ polarization channels. Ice et al. (2014) further point out that to obtain an accurate bias it is essential to point the antenna exactly at the sun. This is because the power difference varies across the solar disk due to the mismatch between the $\mathrm{H}$ and $\mathrm{V}$ antenna beam shapes. This is evident in the scatterplots of solar $Z_{\mathrm{DR}}$, which were derived from operational scanning data, and are similar to those presented by Holleman et al. (2010b) and Huuskonen et al. (2016). Ice et al. (2014) also show examples of operational solar monitoring data, using solar interferences recorded during sunrise and sunset, and discuss their use for long-term monitoring of antenna bias and $Z_{\mathrm{DR}}$. Similarly, Zittel et al. (2014) used solar observations of $Z_{\mathrm{DR}}$ together with observations in light rain and snow to determine the receiver and transmitter path biases.

In this paper, we present a solar monitoring analysis based on one year of level-II data from the NEXRAD radars across the contiguous United States (Ansari et al. 2018). We show case studies of individual radars, as well as statistical outcomes on antenna pointing, received solar flux, beamwidth, and $Z_{\mathrm{DR}}$ throughout the network.

\section{Noise power data}

In previous works, we have detected the solar interferences by searching for radials with a constant signal at long ranges within the operational scanning data. However, in this work we used the radial-by-radial noise powers contained within the NEXRAD operational scan data (level-II metadata). The NEXRAD signal processor applies the method by Ivić et al. (2013) for estimating the noise power as a function of azimuth and elevation and uses these estimates to correct the affected base radar products (i.e., reflectivity factor, spectrum width, differential reflectivity, and copolar correlation coefficient). This method can handle larger noise power disturbances often seen at low elevations, which is important for low-to-medium signalto-noise-ratio (SNR) measurements. The Ivić et al. (2013) method first removes sharp discontinuities and then, in a number of iterative steps, uses flat regions in the radial to estimate the noise power. The solar signal is wide band and hence appears to the signal processor as an additive white Gaussian noise, similar to the thermal noise. Thereby, the solar power is removed from the reflectivity data via the noise subtraction, but it is still available in the noise power estimate.

The WSR-88Ds use, depending on the local settings, different volume coverage patterns (VCPs), which are composed of a varying number of elevation scans recorded with specific waveform types (WFT). Some important properties of the four WFTs and an example of a VCP are shown in Table 1. VCP-212 is a commonly used scan pattern and covers a total of 14 unique elevations between $0.5^{\circ}$ and $19.5^{\circ}$, and is employing various WFTs. Table 1 shows the WFTs, the elevations in the VCP, the azimuthal resolutions, and the number of surveillance and Doppler pulses. For the number of Doppler pulses a value range is given, due to the dependence of the actual settings by the local operator.

WFT1 is designed for contiguous surveillance of reflectivity factor and polarimetric variables at low elevations, using low pulse repetition frequencies. In contrast, WFT2 is optimized for contiguous Doppler measurements (higher pulse repetition frequencies) with ambiguous resolution, also at low elevations. WFT1 and WFT2 are always employed in an alternating mode, i.e., a surveillance scan is followed by a Doppler scan. The radial-by-radial noise powers cannot be accurately determined during WFT2 scans because of their short ambiguous range (i.e., the radial power profile is often saturated with signal returns which prevents accurate noise power estimation). Therefore, the WFT1 noise power estimates are used for both scans in the WSR-88D processing. For this reason, only the WFT1 noise powers are used for the solar monitoring and the WFT2 powers are neglected herein.

Generally WFT1 and WFT2 are recorded in "superresolution" mode where the effective azimuthal sampling spacing is about $0.5^{\circ}$ instead of $1^{\circ}$ (i.e., the expected resolution for a stationary beam whose half power width is $0.95^{\circ}$ ). Super resolution makes a compromise in that the slightly higher standard deviation of the estimates are traded for a gain in resolution. This gain is achieved by applying a von Hann window to the raw level-I data (Torres and Curtis 2007). Even though the sampling spacing in super resolution scans is $0.5^{\circ}$, estimates at each radial are still based on samples covering $1^{\circ}$ in azimuth due to the antenna rotation. For 
polarimetric quantities a modified Hamming window (dubbed Meza window) is used to reduce the large standard deviations of these estimates (Richardson and Ivić 2019). It should be noted that the noise power estimation processing uses a rectangular window for all scans (S. Torres 2019, personal communication).

The intermediate elevations of VCP-212 (those between $1.8^{\circ}$ and $6.4^{\circ}$ ) are measured in batch mode with WFT4, which consists of three surveillance pulses to measure the reflectivity factor and a varying number of Doppler pulses to measure the other parameters. The surveillance pulses are recorded at a lower pulse repetition frequency and as a result the surveillance measurement covers azimuths roughly between $0.1^{\circ}$ and $0.2^{\circ}$. The surveillance pulses are always recorded at the starting edge of the radial. This leads to a bias in the azimuthal pointing of the noise power estimates. This bias can easily be corrected for in the solar processing, by using the number of surveillance and Doppler pulses per radial and the associated pulse repetition frequencies.

At higher elevations, VCP-212 data are recorded in contiguous Doppler mode without ambiguous resolution (WFT3) which provides reflectivity factor, noise powers, and the other (Doppler) measurements from the same antenna position. Scans recorded using this waveform type can be used for solar monitoring without a pointing correction. Last, it is noted that a whole VCP can be recorded either with a short (about $1.6 \mu \mathrm{s}$ ) or a long (about $4.5 \mu \mathrm{s}$ ) transmitter pulse.

For this work, a computer program was written that reads level-II data from the NEXRAD archive and searches through all available elevations and azimuths for radials with an increased noise power. When the radial-by-radial noise power is at least $2.0 \mathrm{~dB}$ higher than the noise level from the calibration (which measures the noise power at high elevation and projects it for use at lower elevations) and the radar antenna is roughly pointing at the sun (i.e., within $5^{\circ}$ and $10^{\circ}$, for the calculated elevation and azimuth, respectively), a potential solar signature is identified in that particular ray. A solar interference is an incidental capture of solar radiation power by the scanning radar antenna. The applied noise power threshold $(2.0 \mathrm{~dB})$ is a trade-off between a large number of identified solar interferences and an effective rejection of genuine background noise. Each solar interference detection is stored with all required metadata, such as the noise powers from the horizontal and vertical receiver channels and their corresponding values to solar flux units, antenna elevation and azimuth, solar elevation and azimuth, radar name, as well as date/time. The metadata for the solar interferences, including those needed for conversion of the noise powers to solar flux units, are retrieved from the level-II data file. Note that the solar flux unit (sfu) is used by astronomers to express the flux density of the radio energy from the sun (as received at Earth), and is defined with units and magnitude of $10^{-22} \mathrm{~W}$ $\left(\mathrm{m}^{2} \mathrm{~Hz}\right)^{-1}$.

More than one year of level-II data from 142 WSR-88Ds were searched for solar interferences. Radars outside the contiguous Unites States were not used to limit the size and processing time of the dataset. The obtained dataset spans 1 September 2018 to 30 September 2019 and contains more than 4.6 million solar interferences (i.e., about 90
TABLE 2. Overview of the collected solar interferences between 1 Sep 2018 and 30 Sep 2019 for 142 weather radars in the NEXRAD network. The number of solar interferences is given depending on the waveform type and the pulse length.

\begin{tabular}{crrr}
\hline \hline Waveform type & Short pulse & Long pulse & \multicolumn{1}{c}{ Total } \\
\hline WFT1 & 2550504 & 91030 & 2641534 \\
WFT3 & 291201 & 30402 & 321603 \\
WFT4 & 1657373 & 0 & 1657373 \\
Total & 4499078 & 121432 & 4620510 \\
\hline
\end{tabular}

interferences per radar per day). Table 2 lists the number of solar interferences depending on the waveform type and the pulse length. Interferences recorded in the short pulse mode, using WFT1 or WFT4, are the most abundant in the dataset. The remainder of this paper presents the analyses of this comprehensive dataset and the results thereof. Note that this method also works for WFT3 and long pulse in principle.

\section{Conversion to solar flux values}

Before conversion to solar flux units, the radial-by-radial noise power is corrected for the background noise power by subtracting the calibration noise power. Subsequently the background-corrected power is divided by receiver gain, the receiver bandwidth, and the effective antenna area (calculated from the wavelength and antenna gain). The receiver gain is the difference between the hardware noise power (as above) and the noise power at the antenna feed, where the latter is derived from the routine receiver calibration. In the metadata, the noise power at the antenna feed includes the matched filter loss (Doviak and Zrnić 1993, chapter 4.4.3), which is not relevant for the solar measurements, and hence is removed to obtain the receiver gain. Apart from the receiver bandwidth, all required metadata are obtained from the levelII data file, with more details described in the Interface Control Document (WSR-88D Radar Operations Center 2018). The receiver bandwidth was set by the radar software to 714 $\mathrm{kHz}$ for short pulse operation. Furthermore, a power correction is made for losses due to the imperfect overlap of the small solar disk and the large main antenna beam pattern and to the averaging (normal rectangular window) of the received power while the radar antenna is scanning in azimuth (Holleman et al. 2010b). Finally, the retrieved solar flux is multiplied by two to correct for observation of the unpolarized solar radiation by a polarization sensitive receiver, either horizontal or vertical.

Figure 1 shows the noise power (upper panel) obtained with the Ivić et al. (2013) method and the derived solar flux units (lower panel) as a function of azimuth at four elevations from the same volume scan. This volume scan was recorded during a sunset in February 2019 by the KBRO weather radar in Brownsville, Texas. The dashed horizontal line in the upper panel indicates the hardware noise level. The upper panel with the noise power data shows a strong and sharp solar signature at around the $252^{\circ}$ azimuth. Apart from that signature the retrieved noise power is rather constant (within $0.5 \mathrm{~dB}$ ) in both azimuth and elevation. 

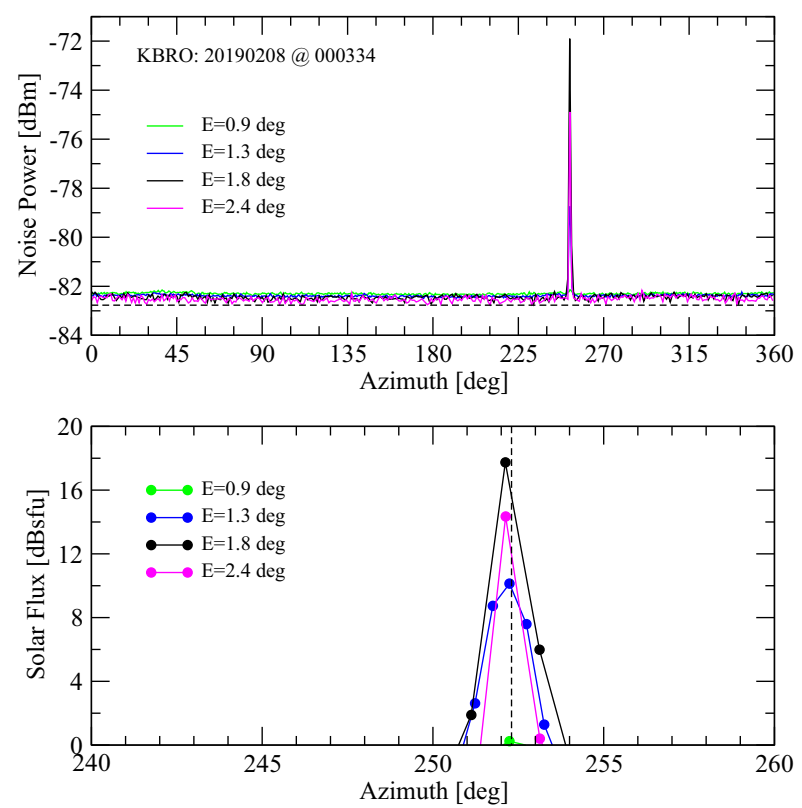

FIG. 1. (top) Noise power and (bottom) derived solar flux as a function of azimuth for the horizontal polarization receiver channel of the KBRO weather radar (Brownsville), recorded on 8 Feb 2019. Curves for different elevations are shown, all recorded during the same volume scan. The dashed horizontal line in the top panel indicates the calibration noise level, $-82.8 \mathrm{dBm}$ in this case, and the vertical dashed line in the bottom panel marks the calculated azimuth of the sun.

The lower panel with the solar flux shows the signature in more detail, with an expanded azimuth scale. Data from each radial can be distinguished and these solar interferences are marked by the dots. The vertical dashed line marks the calculated azimuth of the sun for that particular time and it deviates less than $0.02^{\circ}$ from the observed interferences. The strongest solar hit of $18 \mathrm{dBsfu}$ is observed at an antenna elevation of $1.8^{\circ}$, close to the calculated solar elevation of about $1.6^{\circ}$ for that particular time and location.

In these analyses we use solar interferences from various elevations, including those close to horizon. Hence, the observed elevation and flux need to be corrected for atmospheric refraction (Huuskonen and Holleman 2007) and attenuation (Holleman et al. 2010b). To solve this, we use the analytical formulas for the atmospheric refraction of radio waves and the length of the atmospheric propagation path, which are consistent with the commonly used $k$ model or $4 / 3$ model (Holleman and Huuskonen 2013). As the incoming solar signal tangentially traverses atmosphere, the expected value of $k$ is less than $4 / 3$, which is only valid close to the surface. Hence we use $k=5 / 4$, which fits best to the model calculations and radar observations according to Holleman and Huuskonen (2013). To avoid severe refraction and attenuation, solar interferences observed below $0.9^{\circ}$ elevation are discarded from the analyses.

Figure 2 shows two scatterplots of the solar interferences observed at the KAPX radar (Gaylord, Michigan) between 1

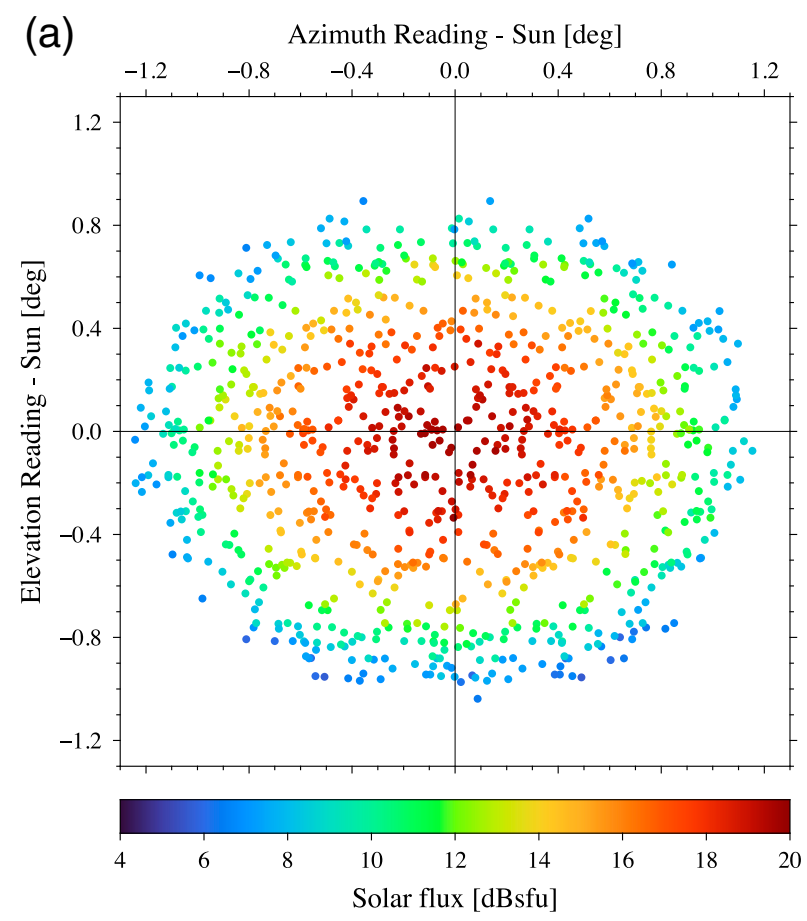

(b)

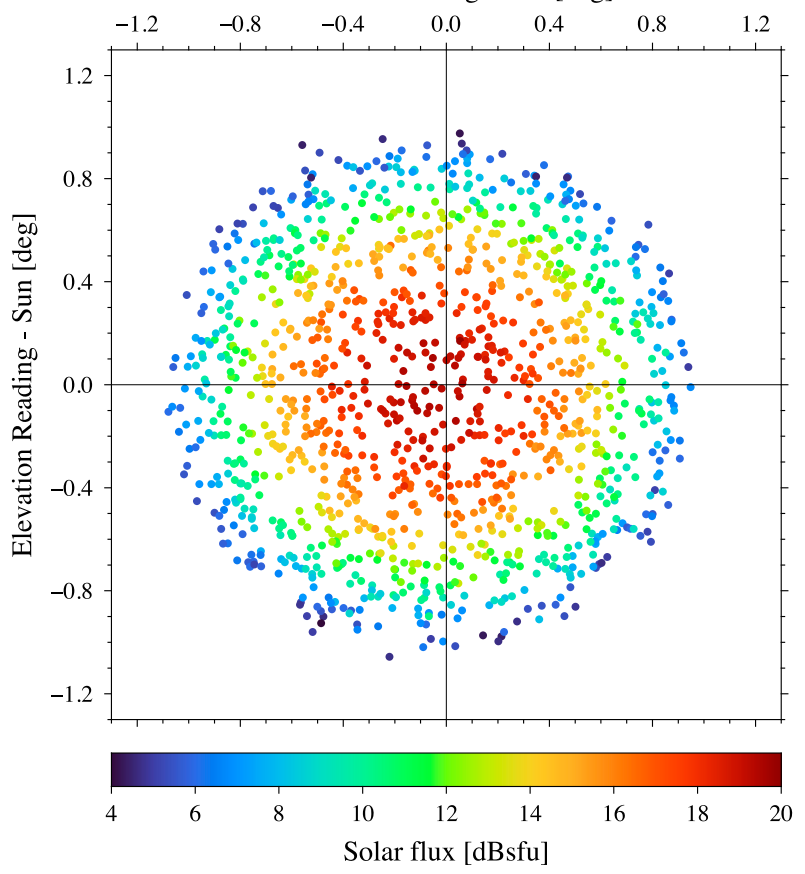

FIG. 2. Two scatterplots of the observed solar interferences by the KAPX radar (Gaylord) between 1 and 31 Jan 2019. (a) The solar interferences observed using WFT1 (940 interferences) and (b) those using WFT4 (1220 interferences), both in short pulse only. The solar flux derived from the noise power received by the horizontal channel is shown. The highest value in both plots is about $19.8 \mathrm{dBsfu}$ while the lowest value is 5.6 and $4.0 \mathrm{dBsfu}$ for WFT1 and WFT4, respectively. 
and 31 January 2019. Figure 2a contains the solar interferences observed by the horizontal channel using WFT1 and Fig. 2b those using WFT4. The distribution is symmetric in both elevation and azimuth, and wider in azimuth than in elevation for WTF1 and roughly spherical symmetric for WFT4. In both panels the strongest solar interferences are found in the center of the plot, where the radar antenna is pointing at the sun. The dynamic range of the observed solar interferences is more than $12 \mathrm{~dB}$, which is higher than for a typical C-band radar (see, e.g., Fig. 2 of Huuskonen et al. 2016). Even though the Sband solar flux of the quiet sun is about $3 \mathrm{~dB}$ lower than that in the $\mathrm{C}$ band (e.g., Benz 2009), the much larger (factor of 4, i.e., about $6 \mathrm{~dB}$ ) effective area of the antenna in NEXRAD makes the dynamic range of the solar interferences larger.

The angular extent of both scatterplots in elevation is about $\pm 0.9^{\circ}$ while that in azimuth is about $\pm 1.2^{\circ}$ and $\pm 0.9^{\circ}$ for WFT1 and WFT4, respectively. It is detailed in section 2 that the azimuthal width is determined by the windows and the number of pulses used in the weather radar signal processing. The solar interferences from WFT1 are averaged over $1^{\circ}$ azimuth while those from WFT4 are based on three azimuth samples only, corresponding to $0.1^{\circ}-0.2^{\circ}$ (see Table 1). For the latter the azimuthal averaging is negligible and thus a circular distribution is expected, which is indeed the case. These twodimensional distributions of the observed interferences are used later for the further analyses of the solar monitoring data.

\section{Analysis of solar flux values}

The formulas for the analysis of the two-dimensional solar hit distributions have been presented in previous publications (Huuskonen and Holleman 2007; Holleman et al. 2010a; Huuskonen et al. 2016), but for convenience we repeat the central formulas here. The distribution of the solar interferences is symmetric in both elevation and azimuth, but usually slightly wider in the latter dimension due to the antenna scanning and integration in azimuth. This results in the effective beamwidth being wider in azimuth than in elevation (Zrnic and Doviak 1976; Torres and Curtis 2007; Huuskonen et al. 2014) as shown in Fig. 2. The received solar power $P_{\text {sun }}$ in dBsfu (i.e., on logarithmic scale) can be modeled by a secondorder polynomial function (Huuskonen and Holleman 2007):

$$
P_{\text {sun }}(x, y) \equiv a_{x} x^{2}+a_{y} y^{2}+b_{x} x+b_{y} y+c,
$$

where $a_{x}$ to $c$ are fit parameters and the coordinates $x$ and $y$ are defined as

$$
\begin{aligned}
& x=\left(\phi_{\text {read }}-\phi_{\text {sun }}\right) \cos \theta_{\text {sun }}, \\
& y=\theta_{\text {read }}-\theta_{\text {sun }}
\end{aligned}
$$

where $\phi$ and $\theta$ denote azimuth and elevation, "read" refers to the angle reading of the radar antenna, and "sun" refers to the calculated solar position. The observed azimuth differences are multiplied by $\cos \theta_{\text {sun }}$ to make them invariant to the elevation (e.g., Doviak and Zrnić 1993, p. 516).
Because Eq. (1) is linear in the fit parameters $a_{x}$ to $c$, the solar inferences data can be easily fitted to this equation by the least squares method. The elevation width $\Delta_{\theta}$, the azimuth width $\Delta_{\phi}$, the elevation bias $B_{\theta}$, the azimuth bias $B_{\phi}$, and the solar peak power when the antenna is pointing directly to the sun, $\hat{P}_{\text {sun }}$, can be calculated from the fit parameters (Huuskonen and Holleman 2007):

$$
\begin{aligned}
& \Delta_{\phi, \theta}^{2}=-\frac{40 \log _{10} 2}{a_{x, y}} \approx-\frac{12}{a_{x, y}}, \\
& B_{\phi, \theta}=-\frac{b_{x, y}}{2 a_{x, y}}, \\
& \hat{P}_{\text {sun }}=c-\frac{b_{x}^{2}}{4 a_{x}}-\frac{b_{y}^{2}}{4 a_{y}} .
\end{aligned}
$$

The widths can only be obtained from Eq. (4) when the corresponding parameter $a_{x, y}$ is negative and otherwise a solution does not exist. It is also noted that we make the assumptions that the angular biases and widths are independent of the pointing angles and that the microwave center of the sun is close to its center of mass (see, e.g., Fig. 5 of Ice et al. 2014).

The WSR-88Ds provide noise powers from both the horizontal and vertical polarization receivers and hence the above fitting procedure is performed independently for both polarizations. An estimate of $Z_{\mathrm{DR}}$ from the sun is obtained from the difference of the horizontal and vertical solar powers. This is different from most radar systems (e.g., those analyzed by Huuskonen et al. 2016), where the radar processor provides the horizontal reflectivity and $Z_{\mathrm{DR}}$, while the vertical reflectivity (and thus the received power) is estimated by subtracting the two quantities.

\section{Monitoring results}

One year of solar monitoring results from the KBMX radar in Birmingham, Alabama, are shown in Fig. 3. These results are obtained by analyzing the solar interferences in intervals of one week. The radar measurements were performed in short pulse mode using either WFT1 or WFT4. These are the most common types of measurements in the NEXRAD network, as can be seen in Table 2. The solar flux measurements from the Dominion Radio Astrophysical Observatory (DRAO) in Kaleden, British Columbia, Canada $\left(49.322^{\circ} \mathrm{N}, 119.620^{\circ} \mathrm{W}\right)$ (Tapping 2013), are also shown as well-known reference values.

The retrieved solar fluxes from the KBMX radar (Fig. 3a) are within $0.1 \mathrm{~dB}$ of those measured at DRAO, which is a really good match. Note that flux deviations from DRAO of up to $1 \mathrm{~dB}$ are reported in Holleman et al. (2010b). The sun was quiet during this period (from 1 September 2018 until 30 September 2019) and hence there are only a few instances where the DRAO solar flux increases from the quiet-sun level, with the strongest events appearing in April and May 2019. These increases are also visible in the solar fluxes retrieved from the radar data. The solar fluxes based on 

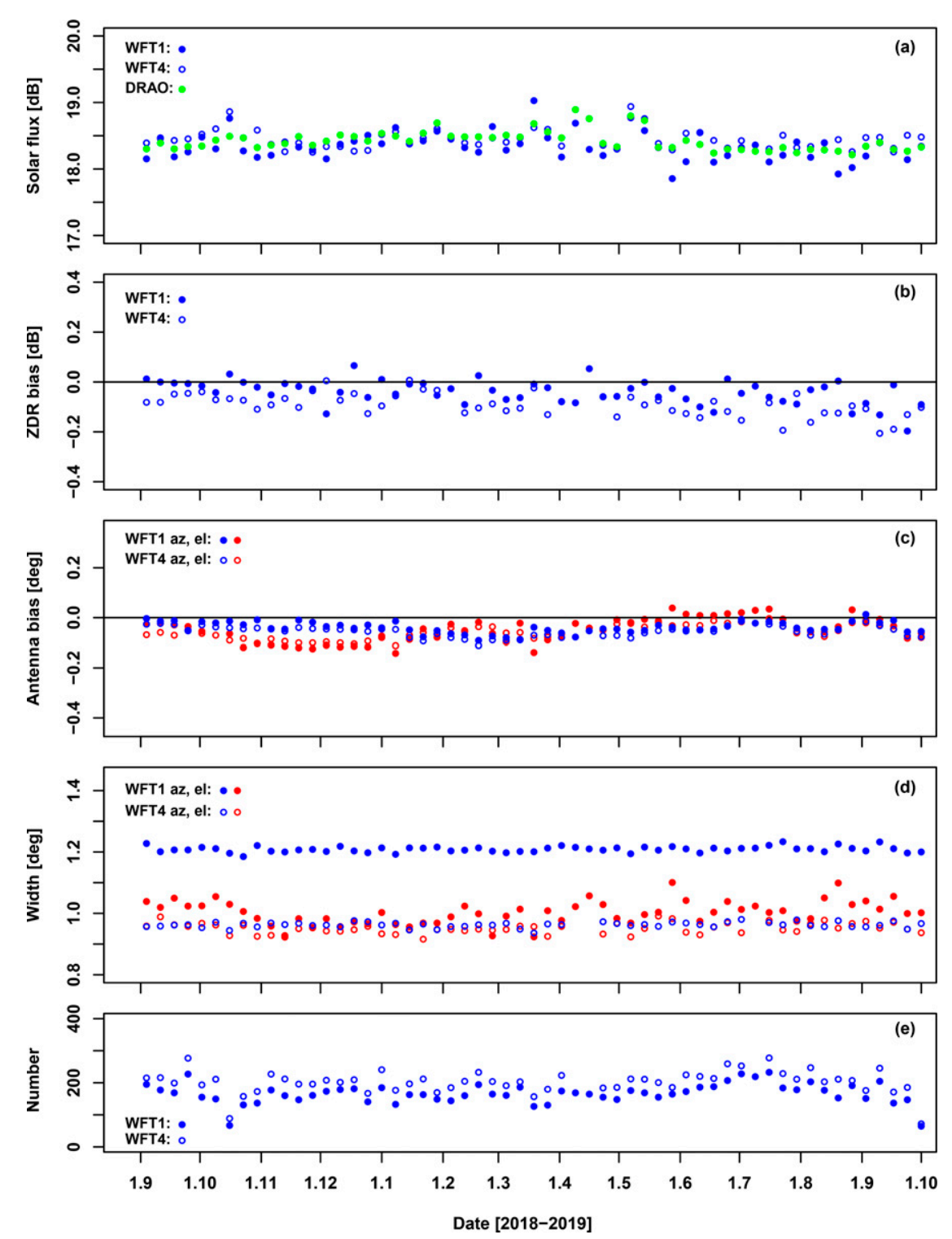

FIG. 3. Results for the KBMX radar from 1 Sep 2018 until 30 Sep 2019 with 7-day integration, based on WFT1 (filled circles) and WFT4 (open circles). (a) Solar flux from data (blue) and from the DRAO observatory (green), (b) difference of flux of horizontal and vertical channels, (c) bias of the antenna azimuth reading (blue) and elevation reading (red), (d) width of the distribution in azimuth (blue) and elevation (red), and (e) the number of solar interferences used in the fit.

WFT1 and WFT4 are closely equal, the difference is less than $0.1 \mathrm{~dB}$. It should be noted that the azimuth windowing on the two datasets is rather different. The WFT1 data are based on collections spanning $1^{\circ}$, whereas the WFT4 data are integrated over $0.1^{\circ}-0.2^{\circ}$. Hence the averaging losses due to the azimuthal scanning, as described by Holleman et al. (2010b), are different for these datasets, the loss being $0.6 \mathrm{~dB}$ larger for WFT1 according to the formulas. The averaging loss has been taken into account in the processing, after which the observed difference between the solar fluxes from the WFT1 and WFT4 data is close to zero, which is a further proof of the validity of the formula by Holleman et al. (2010b).
The $Z_{\mathrm{DR}}$ bias of the sun as observed by the horizontal and vertical channels (receiver path only) of KBMX (Fig. 3b) is slightly negative with a peak-to-peak variation of about 0.2 $\mathrm{dB}$, for both WFT1 and WFT4. Only a few points fall outside this window. The scatter of these points appears larger than that for the solar flux, due to the vertical scale of the plot being smaller. The mean values of the $Z_{\mathrm{DR}}$ bias from WFT1 and WFT4, $-0.04 \pm 0.05 \mathrm{~dB}$, and $-0.09 \pm 0.05 \mathrm{~dB}$, respectively, are overlapping within one standard deviation.

Figure $3 \mathrm{c}$ shows the retrieved elevation and azimuth biases for WFT1 and WFT4 measurements. It is evident that the results for both waveform types are almost equal. It is 

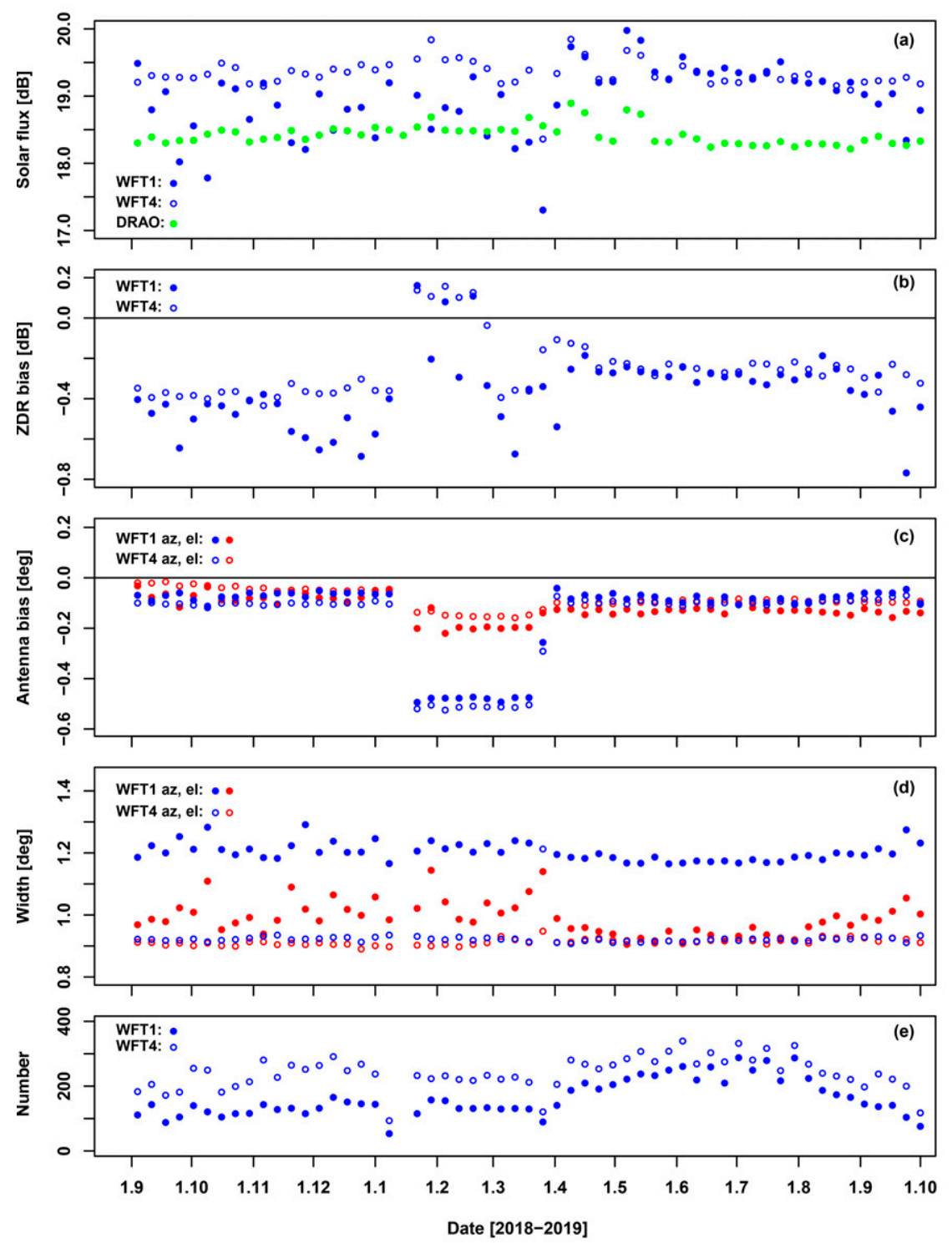

FIG. 4. As in Fig. 3, but for the KRIW radar.

recalled, as detailed in section 2 , that the azimuth data from WFT4 are corrected for the special arrangement of this waveform type. The azimuth bias is rather constant and slightly negative for both WFTs whereas the elevation bias varies between $-0.2^{\circ}$ and $0.0^{\circ}$ during the year.

The elevation and azimuth widths retrieved from WFT1 and WFT4 scans are shown in Fig. 3d demonstrating that the elevation and azimuth widths from WFT1 (filled circles) exceed those from WFT4 (open circles). The elevation width observed by the solar monitoring (red circles) is usually slightly larger than the antenna beamwidth because of the convolution with the solar disk. The average elevation width is $0.95^{\circ}$ for WFT4 and $1.00^{\circ}$ for WFT1. The elevation widths for WFT4 are close to the antenna beamwidth and those of WFT1 are larger and noisier. The latter is attributed to the lower elevations at which WFT1 is employed (see Table 1). At these low elevations more refraction and anomalous propagation are occurring. A much larger difference is observed for the azimuth widths of WFT1 and WFT4. This observation is consistent with the two scatterplots in Fig. 2, for which the azimuth width of WFT1 is also substantially larger than that for WFT4. This is explained by the few surveillance pulses used in the WFT4 scans.

The number of solar interferences (rays with a solar signal) per week varies, but is on the average about 165 for WFT1 and 204 for WFT4. This number depends on receiver sensitivity, scanning strategy, radar location, and season. In this case no clear seasonal variation is observed, but the seasonal variations are expected to be more significant at higher latitudes.

The solar monitoring results do not always look as ideal as those from the KBMX radar. Figure 4 shows the results 
of the weekly analyses for radar KRIW in Riverton, Wyoming. One can divide these results into three periods: 1) from the beginning until mid-January, 2) from mid-January until end of March, and 3) from thereon until the end. During the first period, the retrieved solar fluxes from WFT1 are rather noisy, and the $Z_{\mathrm{DR}}$ bias is substantially negative $(-0.4 \mathrm{~dB})$. During the second period, the elevation and azimuth are stable but far from their expected values and the $Z_{\mathrm{DR}}$ bias is slightly positive. During the third period, the angular biases are set correctly, the $Z_{\mathrm{DR}}$ bias is back to its negative value, and the WFT1 data are more stable. The retrieved solar flux remains too high. The pedestal of radar KRIW was refurbished on 25 March 2019 as part of the NEXRAD Service Life Extension Program (SLEP) and this explains the sudden quality improvement at that day as seen in the monitoring results.

\section{Network results}

The weekly monitoring results of the different quantities can be condensed into single data points by averaging the data over the whole period (i.e., from 1 September 2018 until 30 September 2019). Pairs of these data points for all NEXRAD radars can be displayed in scatterplots. Figure 5 shows such scatterplots for the retrieved elevation and azimuth biases of the antenna. Figure 5a shows the biases obtained from the horizontal and vertical receiver channels using WFT1. Figure 5b shows the biases from the horizontal channel only but recorded using either WFT1 or WFT4. According to these scatterplots the elevation and azimuth pointing accuracy of the radars in the NEXRAD network is generally good, except for two radars having an elevation bias in excess of $-0.2^{\circ}$ (off scale). The observed pointing difference between the horizontal and vertical receiver channels is in most cases below $0.01^{\circ}$ (105 out of 142 radars) and never higher than $0.07^{\circ}$. The differences between the monitoring results from the WFT1 and WFT4 scans are small, suggesting that the azimuth corrections on the WFT4 data (see section 2) are working well. For the whole network, a slightly negative elevation bias of $-0.02^{\circ} \pm 0.08^{\circ}$ is observed (i.e., the antennas are pointing a bit lower), which is fully within requirement.

The slightly negative bias of the azimuth reading $\left(-0.05^{\circ} \pm\right.$ $0.04^{\circ}$ ) is most probably caused by the signal processor algorithms; the radar volume may not be centered exactly toward the nominal center point due to the way the samples are grouped into radials and processed. The distribution of the elevation and azimuth biases is symmetric indicating that these biases are not correlated.

Figure 6 in Huuskonen et al. (2018) shows solar monitoring results of the elevation and azimuth biases from the European OPERA network for March 2018. These monitoring results are shown as histograms and not as scatterplots but a qualitative comparison can be made. The distribution of the elevation biases is quite similar between the two networks, but the distribution of the azimuth biases is much narrower for NEXRAD. This is most likely due to the fact that the NEXRAD network, in contrast to the OPERA network, consists of similar radars (WSR-88D). (a)

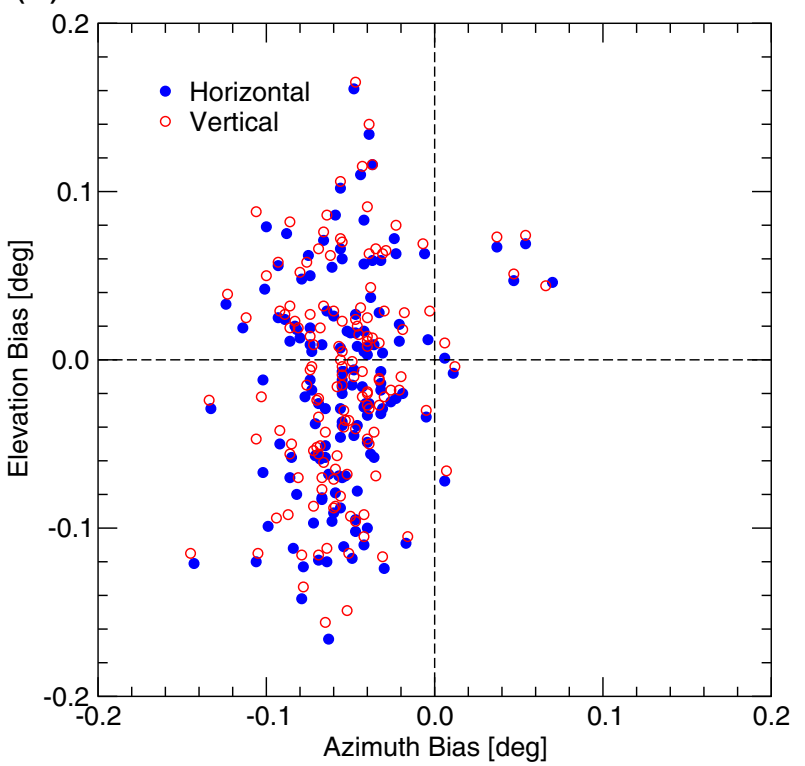

(b)

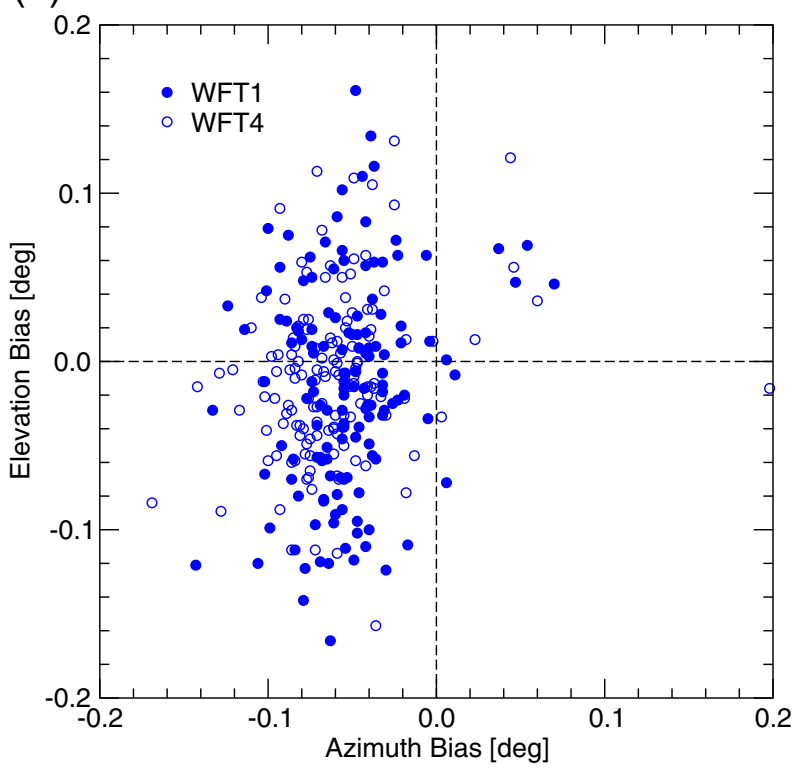

FIG. 5. Scatterplots of the averaged elevation and azimuth biases per weather radar for the whole dataset, i.e., from 1 Sep 2018 until 30 Sep 2019. (a) The biases from the horizontal (blue filled circles) and vertical (red open circles) receiver channels both recorded using WFT1 and short pulse. (b) The biases from the horizontal channel only but recorded using WFT1 (blue filled circles) or WFT4 (blue open circles).

Figure 6 (in this paper) shows scatterplots of the averaged horizontal and vertical polarization solar fluxes per weather radar, for the entire NEXRAD network and for the yearlong dataset. Figure $6 \mathrm{a}$ shows the horizontal and vertical solar fluxes recorded with short pulse using either WFT1 or WFT4. It is evident that the scatter of the retrieved solar fluxes from the horizontal and vertical receiver channels is rather small, 
(a)

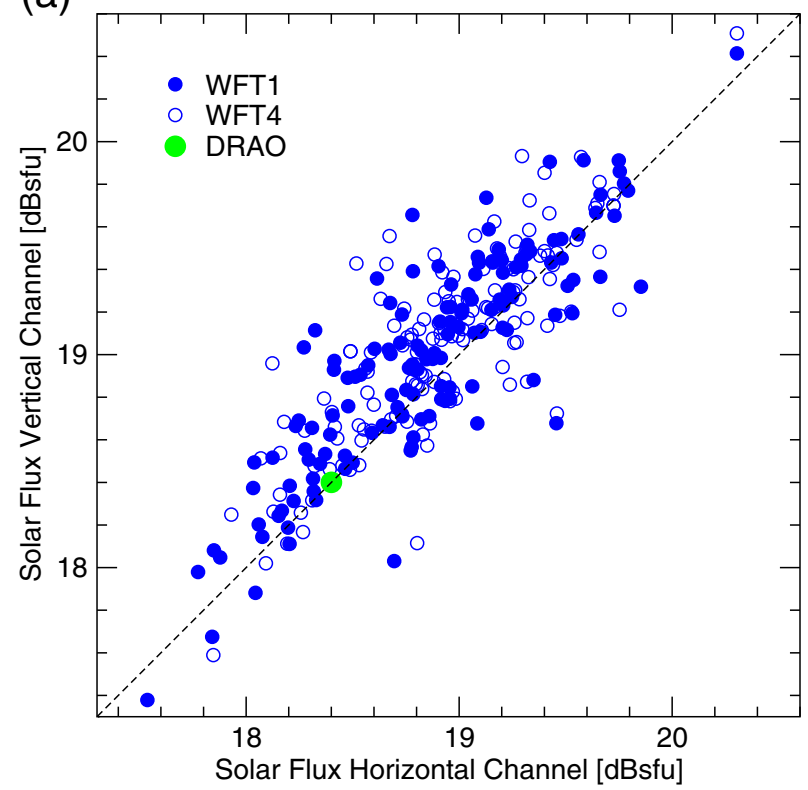

(b)

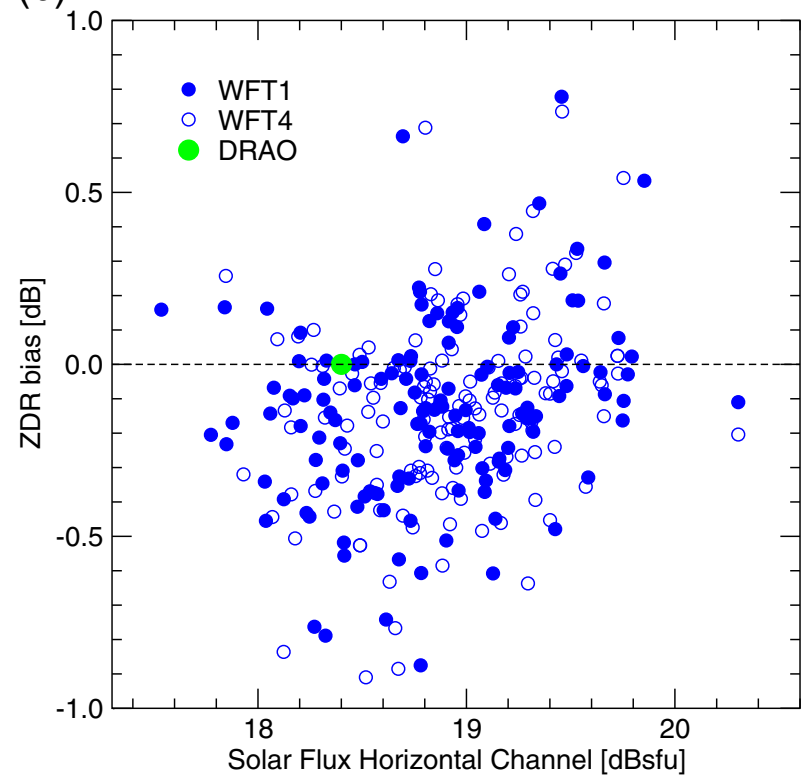

FIG. 6. Scatterplots of the averaged horizontal and vertical polarization solar fluxes per weather radar for the whole dataset, i.e., from 1 Sep 2018 until 30 Sep 2019. (a) The horizontal and vertical solar fluxes recorded using either WFT1 (filled circles) or WFT4 (open circles). The filled green circle represents the solar flux from the DRAO monitoring station averaged over the same period which is $18.4 \mathrm{dBsfu}$. (b) The $Z_{\mathrm{DR}}$ bias as retrieved from the horizontal and vertical solar fluxes using either WFT1 or WFT4.

i.e., about $1 \mathrm{~dB}$. This indicates that the hardware calibration levels of the WSR-88Ds in the NEXRAD network are consistent. However, for most radars the retrieved solar flux is between 0 and $1 \mathrm{~dB}$ above that from the DRAO monitoring station during the same observation period (18.4 dBsfu). It should be noted that the overall calibration of a weather radar is the sum of the receiver (monitored here) and the transmitter calibration.

The receive $Z_{\mathrm{DR}}$ biases retrieved from the horizontal and vertical solar fluxes are shown in Fig. 6b. Both WFT1 and WFT4 cluster around the dashed line marking a zero bias. The network mean value of the system $Z_{\mathrm{DR}}$ bias on receive is calculated to be $-0.13 \pm 0.27 \mathrm{~dB}$ for both WFT1 and WFT4 As this solar method monitors the receiver path of radar systems, there is no fundamental reason for the system $Z_{\mathrm{DR}}$ bias to be close to zero, even though the observed signal is unpolarized. Any deviation from zero in the receiver path is compensated by an opposite deviation in the transmitter path, when a radar system total $Z_{\mathrm{DR}}$ offset is calibrated to zero (see, e.g., Zittel et al. 2014). The scatter of the solar $Z_{\mathrm{DR}}$ biases is rather small suggesting that the horizontal and vertical receiver calibration biases of a radar are correlated.

Another way of looking at the solar monitoring results of the NEXRAD weather radar network is by plotting these results on geographical maps as presented in Fig. 7. These maps display the horizontal solar flux and the retrieved $Z_{\mathrm{DR}}$ bias for all radars (using WFT1 and short pulse) in the continental-scale network. This kind of geographical visualization is ideally suited for a real-time solar monitoring of operational weather radar networks. The observed horizontal polarization fluxes of the sun are consistent over the whole network (mostly green and blue, i.e., between 18 and $19.5 \mathrm{dBsfu}$ ). Two distinct outlying radars are evident, i.e., on the low side the KRTX radar in Portland, Oregon (pink, $17.5 \mathrm{dBsfu}$ ), and on the high side the KOHX radar in Nashville, Tennessee (orange, $20.3 \mathrm{dBsfu}$ ).

The retrieved $Z_{\mathrm{DR}}$ biases, as shown in Fig. $7 \mathrm{~b}$, reveal a rather homogeneous pattern although a slight negative trend from west to east of the continent is suggested (more green dots in the west and more blue in the east). The same trend is seen in the retrieved $Z_{\mathrm{DR}}$ biases from the WFT4 scans (not shown here). We have no explanation for this apparent trend. For the KGJX radar in Grand Junction, Colorado, the lowest $Z_{\mathrm{DR}}$ bias is observed (purple, $-0.88 \mathrm{~dB}$ ) and for the KGWX radar in Columbus, Mississippi, the highest (orange, $0.78 \mathrm{~dB}$ ).

\section{Figure of merit}

The operational performance of the radar network can be assessed by regular visual inspection of the monitoring results or the geographical maps, but an automated assessment is preferred for a large network. To achieve this automation, it is beneficial to define a figure of merit (FoM), which can be calculated from the monitoring results of each radar. We define a FoM of the following simple form:

$$
\begin{aligned}
\mathrm{FoM} & \equiv \sqrt{\frac{1}{N} \sum_{n=1}^{N} s_{n}^{2}}, \\
s_{n} & =\left(\frac{\sigma_{n}}{a_{n}}\right),
\end{aligned}
$$


(a)

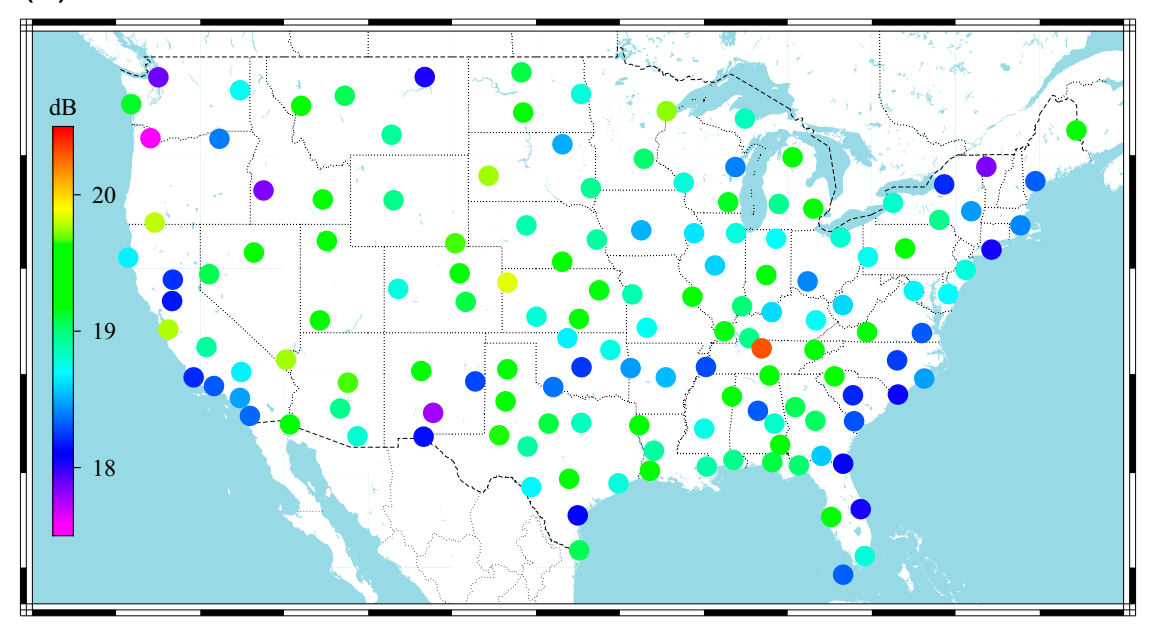

(b)

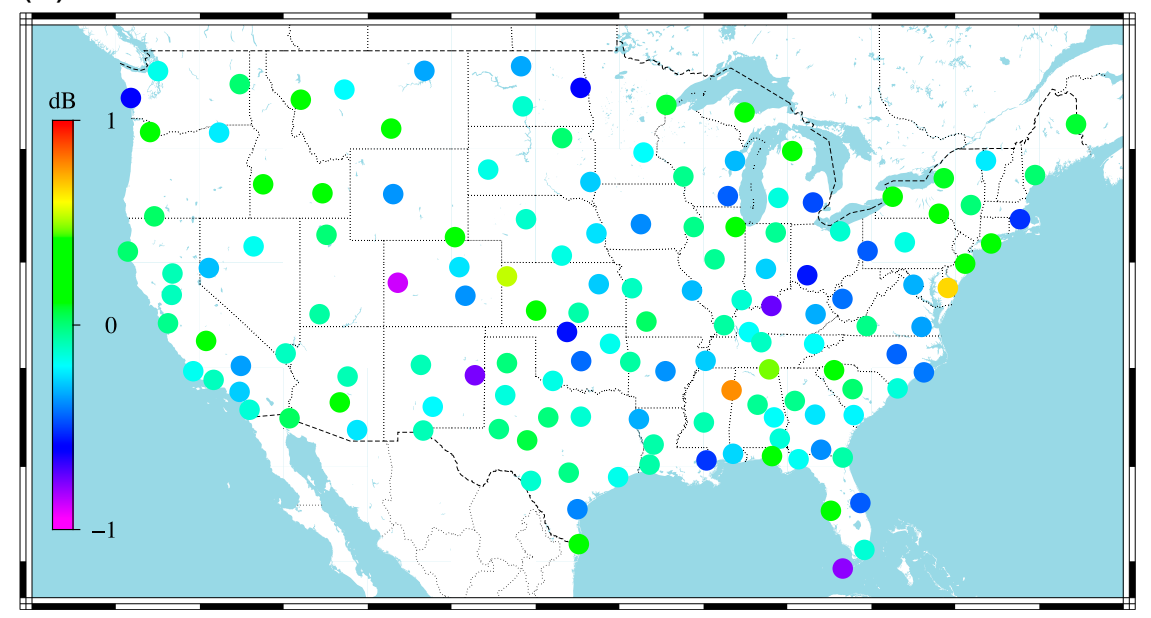

FIG. 7. Geographical maps with the solar flux monitoring results for all NEXRADs and averaged over the whole dataset, i.e., from 1 Sep 2018 until 30 Sep 2019. Results for WFT1 and short pulse are displayed. (a) The horizontal polarization solar fluxes and (b) the retrieved $Z_{\mathrm{DR}}$ biases.

where the dimensionless precision figure $s_{n}$ is calculated for a number $N$ of solar monitoring quantities from the standard deviation $\sigma_{n}$ and the required precision $a_{n}$. The FoM of a radar which on the average meets the set of precision requirements will be one (on average). The standard deviations of the quantities are calculated from the solar monitoring results (e.g., in Figs. 3 and 4). The required precisions for the angular biases, $Z_{\mathrm{DR}}$, and the derived solar flux (requirement for reflectivity is used) are obtained from the WMO Guide (WMO 2018). No requirement is given there for the precision of the angular width values and therefore we have used the value given for the angular biases. The solar monitoring quantities and their required precisions are listed in Table 3.

It should be noted that this FoM is based on the precisions of the radar-hardware quantities and not on their accuracies (i.e., the bias with respect to a reference). The latter would require more radar (reference) parameters and is thus less generic. Moreover, large fluctuations around the sample mean indicate that the required accuracy is not met all of the time, independent of the value of the true mean bias. Poor precision is often caused by hardware malfunctions or imperfections, and hence is an indication of a need for maintenance. In this sense our FoM analysis focuses on required maintenance efforts.

Table 3 shows the calculated distributions of the dimensionless precision figures, i.e., per solar monitoring quantity, for the NEXRAD network between 1 September 2018 and 30 September 2019 for WFT1 and short pulse. For the derived solar flux and azimuth bias, $86 \%$ of the radars have a precision figure of less than 0.5 , meaning that the observed standard deviation for those quantities is a factor of 2 below the required precision. Thus, the requirements for solar flux and azimuth bias 
TABLE 3. Distribution of the dimensionless precision figure $s_{n}$ for the solar monitoring quantities in column 1, with precision requirements $a_{n}$ in column 2 . The range of values of $s_{n}$ in each distribution bin is shown above columns 3-6.

\begin{tabular}{llcccc}
\hline \hline \multicolumn{1}{c}{ Quantity } & \multicolumn{1}{c}{$a_{n}$} & $s_{n}<0.5$ & $0.5 \leq s_{n}<1.0$ & $1.0 \leq s_{n}<2.0$ & 4 \\
\hline Solar flux & $1 \mathrm{~dB}$ & 123 & 15 & 5 & $s_{n} \geq 2.0$ \\
$Z_{\text {DR bias }}$ blevation bias & $0.2 \mathrm{~dB}$ & 105 & 31 & 5 & 0 \\
Azimuth bias & $0.1^{\circ}$ & 92 & 41 & 7 & 4 \\
Elevation width & $0.1^{\circ}$ & 123 & 11 & 7 & 7 \\
Azimuth width & $0.1^{\circ}$ & 117 & 16 & 7 & 4 \\
\hline
\end{tabular}

are met very well by a large majority of the radars. For the $Z_{\mathrm{DR}}$ bias on receive and the elevation bias, this is true for $73 \%$ and $64 \%$ of the radars, respectively, and for the elevation and azimuth widths for about $80 \%$. These results suggest that it is easier to keep the azimuth pointing correct than the elevation pointing. Moreover, it appears that the precision requirement for $Z_{\mathrm{DR}}$ (receiver only) is more difficult to meet than that for the solar flux, which is directly related to the radar reflectivity.

Figure 8 shows the FoM distribution for the radars in the NEXRAD network, calculated from the dimensionless precision figures using Eq. (7). It is evident that a clear majority of the radars, 108 out of 142, have a FoM smaller than 0.50 and that 129 radars have a FoM smaller than one. The best-performing radar according to this measure is KTYX (Montague, New York) with a FoM of 0.21 and the KBMX radar shown in Fig. 3 has an FoM of 0.30 . The monitoring results of the 13 radars with a FoM larger than one have been inspected visually whereby large gaps and fluctuations in the solar monitoring data are observed. These poor monitoring results can be caused either by radar hardware or processing issues or by failure of the solar monitoring, e.g., by lack of good quality interference data.

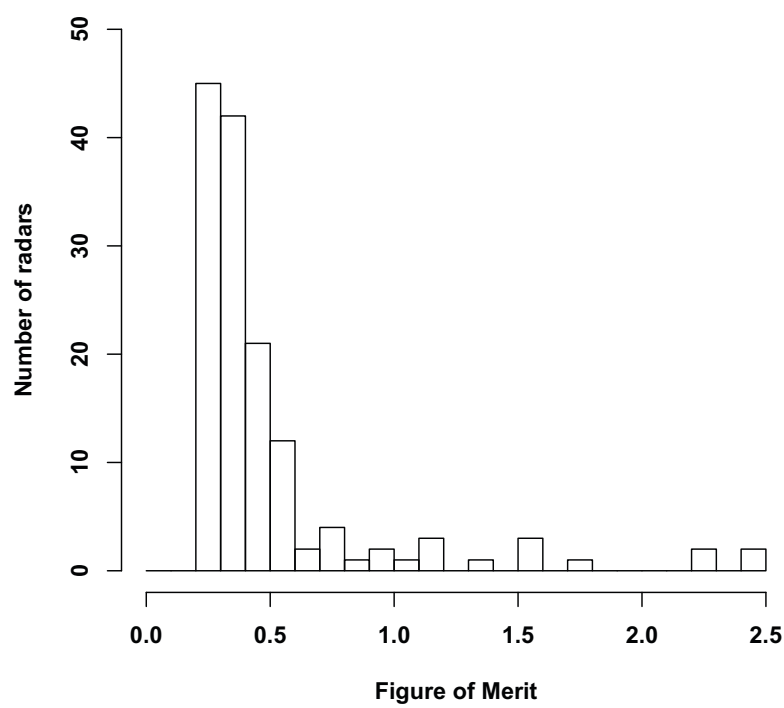

FIG. 8. Distribution of the figure of merit of all radars in the NEXRAD network. The figure of merit is based on the standard deviations of several quantities obtained from the solar monitoring during the period from 1 Sep 2018 until 30 Sep 2019. The bin size is 0.1 .
Our case study of the KRIW radar (see Fig. 4) demonstrates that the analysis results indicate clear quality problems even though the FoM (0.82) is less than one. This indicates that the FoM quality threshold may be set too high. In that regard, the results seen in Fig. 8 suggest using a FoM threshold of 0.6, which is the upper edge of the symmetric distribution. Altogether, 120 radars meet this FoM threshold.

\section{Summary and conclusions}

We have presented results from the analysis of one year of operational solar monitoring data generated by 142 NEXRADs across the contiguous United States. Solar monitoring is a method in which solar interferences, recorded during operational scanning of a radar, are used to monitor antenna pointing, identify signal processor issues, track receiver chain stability and calibration, and check the balance between horizontal and vertical polarization receiver chains. Our dataset spans the period from 1 September 2018 until 30 September 2019 and contains more than 4.6 million solar interferences, which amount to about 90 interferences per radar per day. Case studies, geographical maps, and statistical results on antenna alignment biases, solar fluxes, and $Z_{\mathrm{DR}}$ biases have been presented and discussed.

According to the operational solar monitoring the elevation and azimuth pointing accuracy of the radars in the NEXRAD network is good, with the exception of 2 radars with an elevation bias in excess of $-0.2^{\circ}$. Proper adjustment of the antenna elevation is critical for the radar performance but it is complicated because the physical and electrical pointing of the antenna need to coincide. Meanwhile, very few, if any, reference objects besides the sun are available. For the whole network, a slightly negative elevation bias of $-0.02^{\circ} \pm 0.08^{\circ}$ is observed (i.e., the antennas are pointing a bit lower), which is fully within requirement. We conjecture that the slightly negative bias of the azimuth reading $\left(-0.05^{\circ} \pm 0.04^{\circ}\right)$ is most probably caused by the signal processor algorithms. The observed pointing difference between the horizontal and vertical receiver channels is below $0.01^{\circ}$ for 105 out of the 142 radars.

The $Z_{\mathrm{DR}}$ bias in the receive path, as retrieved from the horizontal and vertical solar fluxes, is scattered around zero, with a network mean at $-0.13 \pm 0.27 \mathrm{~dB}$. As the solar method monitors the receive path of the radar system only, there is no 
fundamental reason for this $Z_{\mathrm{DR}}$ bias to be close to zero, even though the observed signal is unpolarized.

Another way of looking at the solar monitoring results of the NEXRAD network is by plotting these results on geographical maps. This kind of geographical visualization is ideally suited for a real-time solar monitoring of large operational weather radar networks. The observed horizontal polarization fluxes of the sun are fairly consistent over the whole network although a few distinct outliers are observed. The retrieved $Z_{\mathrm{DR}}$ biases reveal a rather homogeneous geographical pattern although a slight negative trend from west to east of the continent is suggested.

The performance of the radar network has been assessed using a figure of merit based on the required precision of the solar monitoring quantities. The assessment per quantity suggests that it is easier to keep the azimuth pointing correct than the elevation pointing and that the precision requirement for $Z_{\mathrm{DR}}$ is far more difficult to meet than that for reflectivity. Results from this work indicate that the majority of the radars (i.e., 120 out of 142) meet the quality requirements.

In conclusion, it is found that the solar monitoring method provides great benefit for monitoring and harmonizing national and transnational operational radar networks.

Acknowledgments. We gratefully acknowledge Walter D. Zittel and Alan D. Free from the National Weather Service Radar Operations Center in Norman, Oklahoma, for their support and fruitful discussions. We also thank the anonymous reviewers who provided us with many good suggestions that improved the quality of the manuscript. Datasets analyzed during the current study are available in github.com/taylbm/scansun_nexrad. These datasets were derived from the following public domain resources: NEXRAD level-II data available at s3.amazonaws.com/noaa-nexradlevel2/index.html.

\section{REFERENCES}

Altube, P., J. Bech, O. Argemí, and T. Rigo, 2015: Quality control of antenna alignment and receiver calibration using the sun: Adaptation to midrange weather radar observations at low elevation angles. J. Atmos. Oceanic Technol., 32, 927-942, https://doi.org/10.1175/JTECH-D-14-00116.1.

Ansari, S., and Coauthors, 2018: Unlocking the potential of NEXRAD data through NOAA's big data partnership. Bull. Amer. Meteor. Soc., 99, 189-204, https://doi.org/10.1175/ BAMS-D-16-0021.1.

Bean, B. R., and E. J. Dutton, 1966: Radio Meteorology. National Bureau of Standards Monogr., No. 92, U.S. Government Printing Office, 435 pp.

Benz, A. O., 2009: Quiet and slowly varying radio emissions of the sun. Solar System, J. Trümper, Ed., Landolt-Börnstein, 80-89.

Crum, T., and T. Alberty, 1993: The WSR-88D and the WSR-88D Operational Support Facility. Bull. Amer. Meteor. Soc., 74, 1669-1687, https://doi.org/10.1175/1520-0477(1993)074<1669: TWATWO $>2.0 . \mathrm{CO} ; 2$.

Doviak, R. J., and D. S. Zrnić, 1993: Doppler Radar and Weather Observations. 2nd ed. Academic Press, 562 pp.
Frech, M., and J. Hubbert, 2020: Monitoring the differential reflectivity and receiver calibration of the German polarimetric weather radar network. Atmos. Meas. Tech., 13, 10511069, https://doi.org/10.5194/amt-13-1051-2020.

Gabella, M., M. Sartori, M. Boscacci, and U. Germann, 2015: Vertical and horizontal polarization observations of slowly varying solar emissions from operational Swiss weather radars. Atmosphere, 6, 50-59, https://doi.org/10.3390/atmos6010050.

Holleman, I., and A. Huuskonen, 2013: Analytical formulas for refraction of radiowaves from exoatmospheric sources. Radio Sci., 48, 226-231, https://doi.org/10.1002/rds.20030.

,,-- R. Gill, and P. Tabary, 2010a: Operational monitoring of radar differential reflectivity using the sun. J. Atmos. Oceanic Technol., 27, 881-887, https://doi.org/10.1175/2010JTECHA1381.1.

,,-- M. Kurri, and H. Beekhuis, 2010b: Operational monitoring of weather radar receiving chain using the sun. $J$. Atmos. Oceanic Technol., 27, 159-166, https://doi.org/10.1175/ 2009JTECHA1213.1.

Huuskonen, A., and I. Holleman, 2007: Determining weather radar antenna pointing using signals detected from the sun at low antenna elevations. J. Atmos. Oceanic Technol., 24, 476-483, https://doi.org/10.1175/JTECH1978.1.

- M. Kurri, H. Hohti, H. Beekhuis, H. Leijnse, and I. Holleman, 2014: Radar performance monitoring using the angular width of the solar image. J. Atmos. Oceanic Technol., 31, 1704-1712, https://doi.org/10.1175/JTECH-D-13-00246.1.

,-- , and I. Holleman, 2016: Improved analysis of solar signals for differential reflectivity monitoring. Atmos. Meas. Tech., 9, 3183-3192, https://doi.org/10.5194/amt-9-3183-2016.

— improving the homogeneity of the European radar network. 10th European Conf. on Radar in Meteorology and Hydrology, Wageningen University and Research, 594-600, https:// doi.org/10.18174/454537.

Ice, R. L., J. G. Cunningham, A. K. Heck, W. D. Zittel, and R. R. Lee, 2014: Polarimetric weather radar antenna calibration using antenna scans. Proc. 36th Annual Symp., Tucson, AZ, Antenna Measurement Techniques Association, 367-372.

Ivić, I. R., C. Curtis, and S. M. Torres, 2013: Radial-based noise power estimation for weather radars. J. Atmos. Oceanic Technol., 30, 2737-2753, https://doi.org/10.1175/JTECH-D-13-00008.1.

Richardson, L. M., and I. R. Ivić, 2019: A less-tapered signal processing window for polarimetric variables. 39th Int. Conf. on Radar Meteorology, Nara, Japan, Amer. Meteor. Soc., 3-20.

Saxion, D. S., and Coauthors, 2011: New science for the WSR88D: Validating the dual polarization upgrade. 27th Int. Conf. on Interactive Information Systems Processing for Meteorology, Oceanography, and Hydrology, Seattle, WA, Amer. Meteor. Soc., 365, https://ams.confex.com/ams/91Annual/webprogram/ Paper183657.html.

Serafin, R. J., and J. W. Wilson, 2000: Operational weather radar in the United States: Progress and opportunity. Bull. Amer. Meteor. Soc., 81, 501-518, https://doi.org/10.1175/15200477(2000)081<0501:OWRITU>2.3.CO;2.

Tapping, K. F., 2013: The $10.7 \mathrm{~cm}$ solar radio flux $\left(F_{10.7}\right)$. Space Wea., 11, 394-406, https://doi.org/10.1002/swe.20064.

Torres, S., and C. Curtis, 2007: Initial implementation of superresolution data on the NEXRAD network. 23rd Conf. on Int. Interactive Information and Processing Systems for Meteorology, Oceanography, and Hydrology, San Antonio, TX, Amer. Meteor. Soc., 5B.10, http://ams.confex.com/ams/ 87ANNUAL/techprogram/paper_116240.htm. 
WMO, 2018: Guide to meteorological instruments and methods of observation: Volume III-Observing systems. WMO Doc. 8, 410 pp., https://library.wmo.int/doc_num.php?explnum_id=9872.

WSR-88D Radar Operations Center, 2018: Interface control document for the RDA/RPG. NWS Doc. 2620002R, RDA build 18.0, 135 pp., https://www.roc.noaa.gov/wsr88d/PublicDocs/ ICDs/2620002R.pdf.

Zittel, W. D., J. G. Cunningham, R. R. Lee, L. M. Richardson, R. L. Ice, and V. Melnikov, 2014: Use of hydrometeors,
Bragg scatter, and sun spikes to determine system $\mathrm{Z}_{\mathrm{DR}}$ biases in the WSR-88D fleet. Eighth European Conf. of Radar Meteorology and Hydrology, Garmisch-Partenkirchen, Germany, DWD and DLR, DAC.P12, https:// www.roc.noaa.gov/WSR88D/PublicDocs/Publications/132_ Zittel.pdf.

Zrnić, D. S., and R. Doviak, 1976: Effective antenna pattern of scanning radars. IEEE Trans. Aerosp. Electron. Syst., AES-12, 551-555, https://doi.org/10.1109/TAES.1976.308254. 